\title{
ACUTE DEPOSITION OF CALGIUM NEAR THE ELBOW
}

\author{
E. S. R. Hughes, Melbourne, Australia
}

From the Royal Melbourne Hospital, Victoria, Australia

Deposits of calcium in the common extensor origin of the forearm as described in three cases in this paper are unusual; only nine similar examples have been found in the literature. The main interest lies, however, not so much in the rarity of the lesion as in the clue it may provide to the etiological problems of "tennis elbow." The characteristic symptoms are indistinguishable from those of tennis elbow; there is acute onset of pain, tenderness and swelling in the region of the lateral epicondyle; and radiographs show a dense shadow below the epicondyle. In patients treated by early operation it is evident that this shadow is due to the deposit of a viscous fluid, consisting largely of calcium phosphate, in the origin of the common extensor aponeurosis. With conservative treatment the fluid may absorb rapidly and the acute symptoms may subside, but the more chronic features of tennis elbow tend to persist.

\section{REVIEW OF THE LITERATURE}

Schmitt (1921) described such a condition in a woman of thirty-four years with typical radiographic appearances. At operation a collection of doughy material was found deep to the origin of the common extensor muscles. It was shown that the deposit consisted of calcium phosphate and calcium carbonate. Carp (1932) reported five cases. In the first, in a man aged fifty-two years, operation eight weeks after the onset of symptoms disclosed a cavity containing a milky fluid deep to the common tendon of origin of the extensors of the forearm. Microscopically the walls of the space showed chronic inflammation with a few calcareous deposits. In the second patient, a woman aged twenty-five years, conservative treatment was adopted, and within four weeks the symptoms subsided and radiographs showed that the opaque shadow had disappeared. Carp attributed this result to rupture of the wall of the cyst by massage. Two other cases showed similarly acute clinical features and the same spontaneous recovery, but the radiographic shadow had a linear outline like a bony spur. In Carp's fifth case the opacity was small and oval in shape; it had not altered significantly after ten weeks. As the symptoms were chronic and unassociated with swelling the case is not strictly comparable with those now under discussion.

Fiorentini (1937) described four typical cases. Three patients were treated by irradiation, with indifferent results. The fourth was treated by incision through the common extensor tendon; a creamy fluid escaped and the cavity was curetted. Hamilton (1938) reported a patient who was treated by immobilisation for six weeks by which time the symptoms had almost subsided and radiographic examination was negative. The case reported by Young (1944) was treated by irradiation; after six weeks there was evidence of some improvement.

\section{CASE RECORDS}

Case 1, female, aged 30 years, typist-Seen on August 25, 1947. Two weeks previously, without apparent cause, there was aching in the left elbow on its lateral aspect. The pain was aggravated by movement of the forearm; it radiated to the dorsum of the hand and fingers. Two days before attending hospital the painful area became swollen and very tender. The pain was increased by pronation and supination of the forearm and by extension of the fingers.

On examination-There was a localised and tender swelling just below the epicondyle (Fig. 1). The overlying skin was warm and oedematous. The elbow was held in flexion. Passive movement was from 170 to 60 degrees. Radiographs showed normal bone contours but there were irregular opacities on the lateral aspect of the joint in close relation to the humerus and radius (Fig. 2). There was no pyrexia. The blood count and erythrocyte sedimentation rate were normal. The Wassermann reaction was negative. 


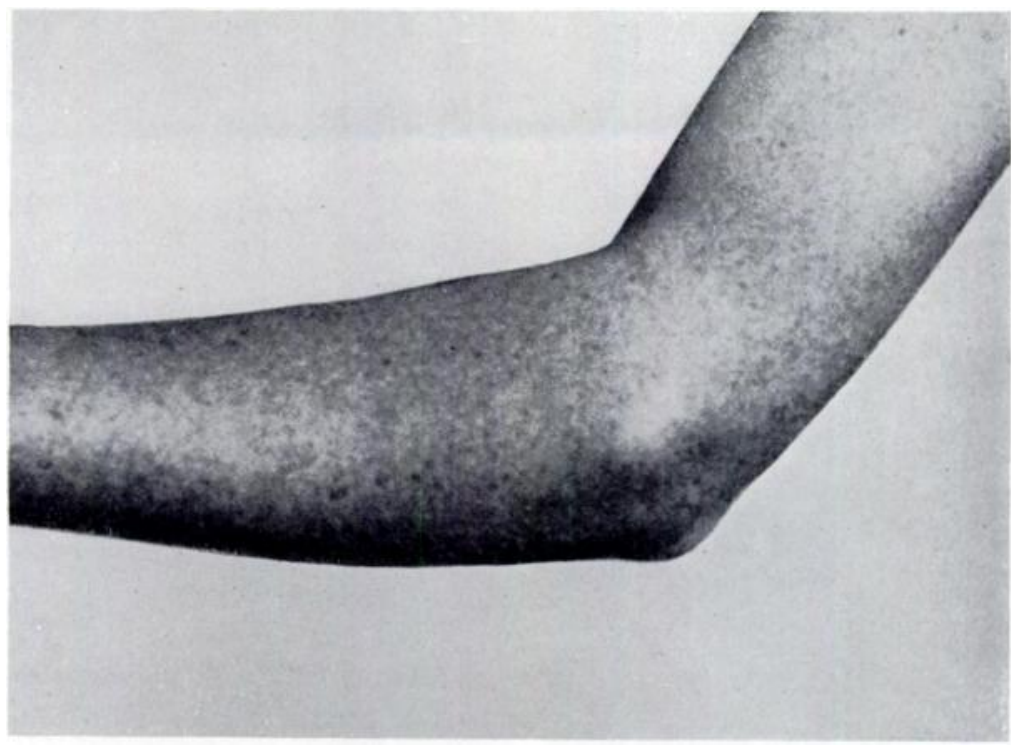

FIG. 1

Case 1. Calcification of the common extensor origin. The local swelling is seen clearly:

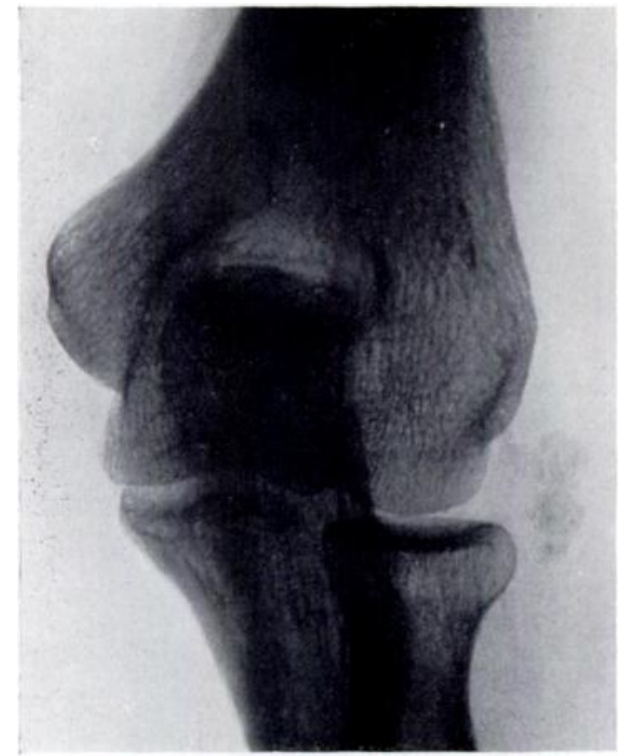

FIG. 2

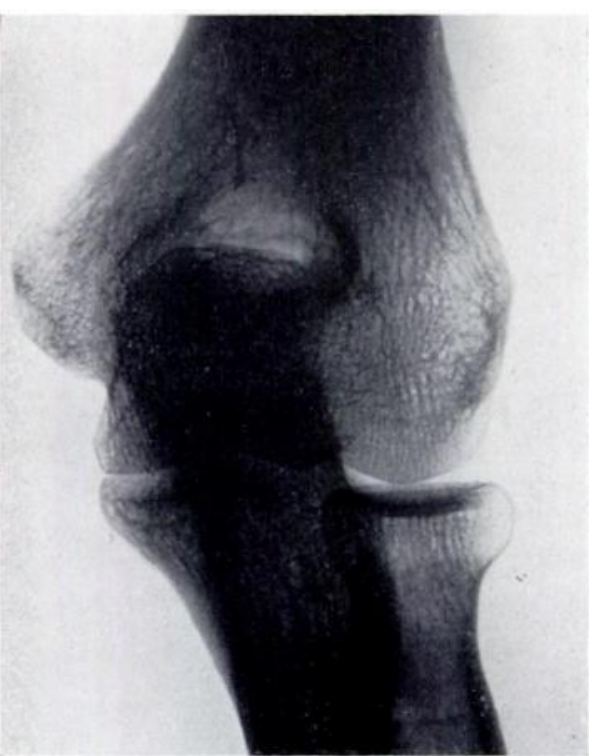

FIG. 3

Case 1. Radiographs at the time of the acute exacerbation show a well-defined area of calcification (Fig. 2). This gradually disappeared under treatment by rest. Figure 3 shows condition at two weeks.

Rest in a sling was advised. After two weeks there was less tenderness and radiographs showed that the density of the opacities was reduced (Fig. 3). Two weeks later the shadows had almost disappeared, and on October 6, six weeks after her first attendance, they could no longer be seen. Nevertheless she still complained of pain and tenderness which persisted with varying severity for many months. Operative treatment was therefore advised.

Operation, March 19, 1948-The common extensor tendon was exposed by a longitudinal incision and divided in the direction of its tendinous fibres 1 centimetre behind the posterior border of the extensor carpi radialis brevis. The subtendinous space between the common tendon and the capsule of the radio-humeral joint was found to be filled with fibrous tissue. This was removed. Within three weeks of operation the symptoms subsided completely and six months later there was no evidence of recurrence.

vol. $32 \mathrm{~B}$, No. 1 , FEBRUARY 1950 
Case 2, female, aged 29 years-Seen on November 12,1947, complaining of aching in the region of the lateral epicondyle of the left elbow for eight months. There was no history of injury. The pain radiated down the back of the forearm to the fingers and it was increased by use of the limb. Three days before hospital attendance the arm became acutely painful and stiff, and a swelling appeared on the lateral aspect of the elbow.

Clinical examination-There was diffuse swelling on the lateral aspect of the elbow. The greatest tenderness was immediately below the lateral epicondyle. There was a painless range of movement from 135 to 80 degrees. Pronation and supination were normal. Extension of the middle three digits against gravity and resistance was painful. Radiographs showed an irregular opacity on the lateral aspect of the joint in close relation to the humerus and radius (Fig. 4). There was no pyrexia. The erythrocyte sedimentation rate and blood count were normal. The blood Wassermann reaction was negative.

Operation, November 19, 1947-The tendon of origin of the common forearm extensors was divided in the line of its fibres; a white, opaque, viscous fluid escaped. Examination showed that it occupied a space between the aponeurosis and the capsule of the radio-humeral joint, extending distally from the epicondyle for a distance of 2 centimetres, and anteriorly and posteriorly to the limits of the aponeurosis. Its walls were coated with a white chalky material; the capacity was about 3 cubic centimetres. After evacuation of all the fluid and curettage of the carity, the skin was closed with interrupted sutures. There was

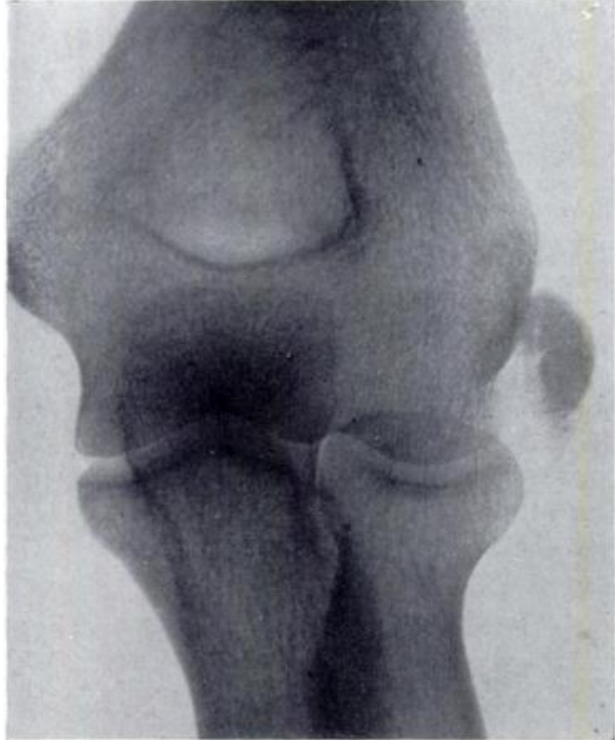

FIG. 4

Case 2. Radiographic appearance at the time of acute exacerbation.

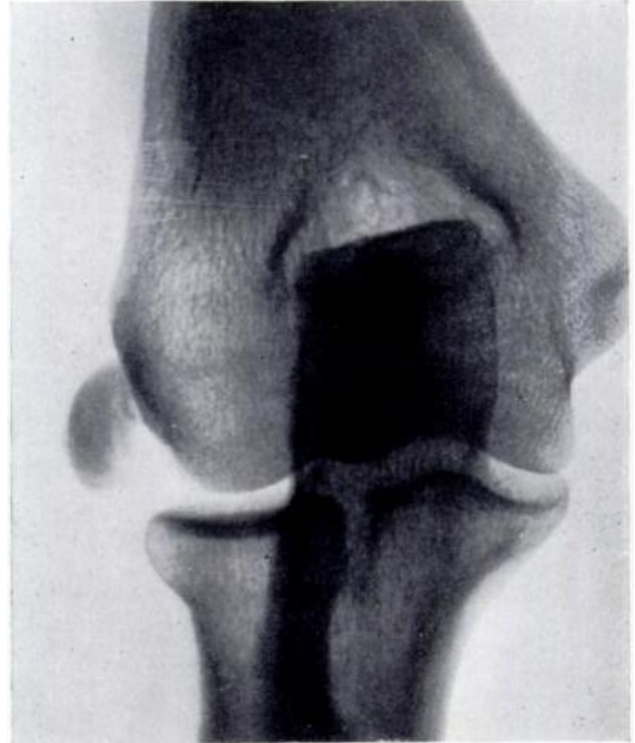

FIG. 5

Case 3. Appearance of the calcified deposit shortly before operation.

immediate relief of symptoms and within three weeks normal function was restored. A year later the left arm differed in no way from the right.

Pathological investigation-Histological examination showed that the wall of the cyst was fibrous, partly necrotic, and infiltrated with calcium salts. There were many lymphocytes and histiocytes. Bacteriological cultures were sterile. Biochemical examination showed that the deposit was largely calcium and phosphate with a little fat and protein (21.3 milligrams calcium, 7.7 milligrams phosphate determined as phosphorus, $34 \cdot 0$ milligrams fat).

Case 3, female, aged 28 years --Seen and treated by Sir Reginald Watson-Jones-Six months' history of pain over the lateral aspect of the right elbow, aggravated by gripping in the right hand and by resisted movements of the forearm. No history of injury.

On examination there was tenderness and slight swelling localised accurately to the common extensor origin immediately below the lateral epicondyle. Radiographic examination showed a calcified deposit on the region of the common extensor origin (Fig. 5). The left elbow and the shoulders were normal.

Operation, July 31, 1942-The common extensor origin was exposed. Within the tendinous fibres there was a yellow-white deposit which was evacuated. It had exactly the same consistency as calcified deposits in the supraspinatus tendon.

Subsequent progress-The pain was at once relieved and after the wound healed full elbow movements were regained within three weeks. Recovery was complete. 


\section{DISCUSSION}

It was considered by Schmitt that there had been a deposit of calcium phosphate in the walls and lumen of a bursa and this view received the support of Carp, Hamilton and Young. Fiorentini was unable to decide whether the lesion was primarily bursal or tendinous. Between the common tendon of origin of the forearm extensors and the capsule of the radio-humeral joint is a tissue space, bounded proximally by the lateral epicondyle, anteriorly by the origin of the extensor carpi radialis brevis muscle, and posteriorly by the insertion of the anconeus muscle. Normally, this space is occupied by loose areolar tissue. In a series of sixteen operations performed for chronic "tennis elbow " no macroscopic or microscopic abnormality was seen in the common tendon of the forearm extensors. In two cases the space under this tendon contained a small thick-walled bursal sac (Fig. 6); in the others it was obliterated by tissue which microscopically resembled the pannus found in larger bursae and in joints. Removal of this tissue afforded immediate and apparently permanent relief. These observations are in close agreement with those of Osgood (1922).

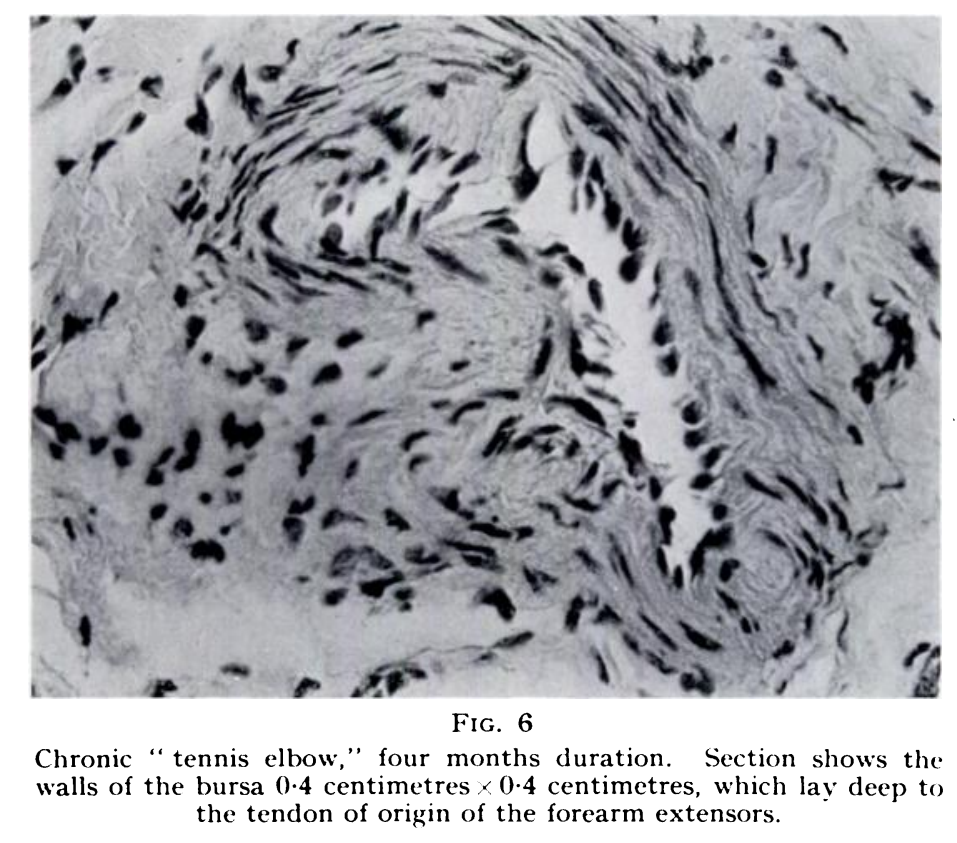

The condition now described resembles very closely that which involves the insertion of the supraspinatus tendon. In both there is an acute attack of pain with limitation of movement superimposed upon a preceding chronic stage and radiographic evidence of a dense irregular shadow. Treated conservatively, the deposit may gradually absorb with corresponding relief of pain. Operation in each condition reveals a deposit of thick opaque fluid. Biochemical analysis in the cases now reported showed calcium and phosphate in proportions comparable to that found by Elmslie (1932) in material removed from the supraspinatus tendon.

Whatever treatment is adopted for calcification in the region of the elbow, whether by rest, irradiation or massage, the deposit seems to be absorbed rapidly; the acute exacerbation subsides and sometimes even chronic symptoms may be relieved. Nevertheless conservative treatment is time-consuming and there is often persistent discomfort. Operation has proved successful and it is the treatment of choice. All that is necessary is to incise the tendon of origin of the common forearm extensors in the line of its fibres, remove the calcified material, curette the walls of the cavity and encourage active exercises after operation.

vol. $32 \mathrm{~B}$, No. 1, FEBRUARY 1950

$\mathrm{C}$ 


\section{SUMMARY}

1. Three cases of localised deposition of calcium salts deep to the origin of the common extensors of the forearm with acute symptoms clinically indistinguishable from " tennis elbow" are described.

2. Reports of nine similar cases have been found in the literature.

3. It is suggested that " tennis elbow " is caused by a lesion, probably an adventitial bursa, in the tissue space between the tendon of origin of the forearm extensors and the capsule of the radio-humeral joint; and that it is the sudden precipitation of calcium phosphate at this site which causes the lesion here described.

4. Acute calcification near the elbow joint is compared with the similar calcification which may occur in the insertion of the supraspinatus tendon.

5. Operative treatment is advised.

I am indebted to Mr Charles Gray for his permission to treat and publish Case 2 and for his helpful criticisms of this paper. Case 3 is published by kind permission of Sir Reginald Watson-Jones, to whom my thanks are due. I wish to thank Dr C. V. Harrison for examining the operation specimens.

\section{REFERENCES}

CARP, L. (1932) : Tennis Elbow (epicondylitis) caused by radio-humeral bursitis. Archives of Surgery, $24,905$. ElmSLie, R. C. (1932): Calcareous deposits in the supraspinatus tendon. British Journal of Surgery, $20,190$. Fiorentini, A. (1937): La periartrite calcarea del gomito. Archivo di Ortopedia, 53, 385.

Hamilton, A. R. (1938): A case of calcification in the epicondylar bursa of the elbow. British Journal of Surgery, 26, 446.

OsGooD, R. B. (1922): Radio-humeral bursitis, epicondylitis, epicondylalgia (Tennis elbow). Archives of Surgery, 4, 420.

Sснміт, J. (1921): Bursitis calcarea am epicondylus externus humeri. Archiv für Orthopädische und Unfall-Chirurgie, 19, 215.

Young, H. H. (1944): Calcified bursitis. Proceedings of the Staff Meetings of the Mayo Clinic, 19, 250. 